\title{
„This present crisis“. Zur Semantik der Krise in der politischen Publizistik Großbritanniens im 18. Jahrhundert
}

Von André Krischer

\section{Einleitung}

Als Reinhart Koselleck ,Kritik und Krise’ Ende der 1950er Jahre verfasste, ging er noch davon aus, „daß dem 18. Jahrhundert der Zusammenhang zwischen der ausgeübten Kritik und der heraufkommenden Krise entging - ein wörtliches Zeugnis für das Bewusstsein des Zusammenhangs ließe sich nicht finden“"1. Erst mit Rousseau und am Ende des Ancien Régime sei Krise im modernen Sinne, nämlich als prognostische Zeitdiagnose, in die politisch-soziale Semantik eingegangen ${ }^{2}$. Seine eigentliche diskursive Virulenz habe der Begriff daher erst im 19. Jahrhundert entfalten können. In späteren Artikeln hat Koselleck diesen Befund allerdings modifiziert: In den ,Geschichtlichen Grundbegriffen’ wies er etwa darauf hin, dass der Begriff Chrysis zumindest in England schon zu Beginn des 17.

Jahrhunderts in einem politischen Sinne gebraucht worden sei, nämlich zur Beschreibung der Situation des Parlaments im Konflikt mit dem König ${ }^{3}$. 1714 habe dann der Publizist Richard Steele zum ersten Mal ein Pamphlet mit, The Crisis’ betitelt ${ }^{4}$. Welche Gründe es für diese semantische Vorzeitigkeit des Begriffs im Englischen gab und welche Folgen dies für den politischen Diskurs in Großbritannien vor 1800 hatte, darüber hat Koselleck allerdings nicht mehr gearbeitet ${ }^{5}$. Mein Beitrag knüpft zwar an diese begriffsgeschichtliche ,Lücke’ an, doch verstehe ich ihn nicht einfach als eine Art Nachtrag. Es soll vielmehr darum gehen, die historische Semantik von Crisis im britischen 18. Jahrhundert mit den Fragestellungen des Konstanzer Zeitdiagnosen-Projekts zu verbinden ${ }^{6}$. Es soll daher um die exemplarische Untersuchung der Krisensemantik im historischen Zusammenhang ebenso gehen wie um die Frage, welche Medien und Diskurse dem Krisenbegriff zum Durchbruch, sogar zur

\footnotetext{
${ }^{1}$ Reinhart Koselleck, Kritik und Krise. Eine Studie zur Pathogenese der bürgerlichen Welt, Frankfurt a.M., 7. Aufl., 1992 [zuerst Freiburg / Müchen 1959], 5.

${ }^{2}$ Ebd., $132 \mathrm{ff}$.

${ }^{3}$ Vgl. dazu in diesem Band den Beitrag von Andreas Pećar.

${ }^{4}$ Sir Richard Steele, The crisis: or, a discourse representing, from the most authentick records, the just causes of the late happy revolution: [...] with some seasonable remarks on the danger of a Popish successor. By Richard, London 1713 [1714].

${ }^{5}$ Reinhart Koselleck, Art. „Krise“, in: Historisches Wörterbuch der Philosophie, Bd. 4, Darmstadt 1976, 12351240, hier 1236, weist allerdings darauf hin, dass ,Crisis’ seit dem 18. Jahrhundert „ein geläufiger Titel polemischer Schriften geworden“" war.

${ }^{6}$ Vgl. die Konferenzankündigung in: [http://hsozkult.geschichte.hu-berlin.de/termine/id=7491].
} 
Popularität verhalfen. Ich werde nun in einem ersten Schritt zunächst zeigen, in welchen historischen Kontexten überhaupt mit dem Krisen-Begriff operiert wurde, um dann das weitere Vorgehen zu skizzieren.

\section{Die historische Semantik der Krise 1700-1800 - eine quantitative und kontextualisierende Annäherung}

Unterteilt man das 18. Jahrhundert in Jahrzehnte und fragt man nach der Häufigkeit des

Vorkommens des Begriffs Krise im politischen Schrifttum in dieser Zeit, dann zeigt sich eine kontinuierliche und schließlich sprunghafte Zuwachsrate ${ }^{7}$. Diese ist allerdings auch damit zu erklären, dass die Publikationsdichte als solche stark zunahm. Zwischen 1700 und 1710 wiesen jedenfalls rund 100 selbständig publizierte Texte den Begriff Krise im zeitdiagnostischen Sinn auf, 1711-20 waren es bereits 225, 1721-30: 150 und 1731-40: 200. Nach dieser ,Stagnation' rekurrierten in den beiden Jahrzehnten zwischen 1740 und 1760 jeweils rund 450 Texte auf den Krisenbegriff, 1761-70: 650, 1771-80: 900, 1781-90: 1110 und schließlich 1791-1800 genau 2675.

Welche historischen Kontexte führten im 18. Jahrhundert nun zur Verwendung des Begriffs Crisis? Wie erwähnt, muss man die Etymologie des Begriffs im figurativen Sinn, nämlich zur Qualifizierung einer politisch-sozialen Situation, im Englischen ins 17. Jahrhundert zurückverfolgen $^{8}$. 1628 brachte damit der Abgeordnete und Dichter Sir Benjamin Rudyerd (1572-1658) seine Sicht auf die Lage des Parlaments auf den Punkt: „This is the Chrysis of Parliaments; we shall know by this if Parliaments live or die” ${ }^{9}$. Man kann davon ausgehen, dass Crisis nicht etwa zufällig aus den naturwissenschaftlich-astrologischen Kontexten ${ }^{10}$ in

\footnotetext{
${ }^{7}$ Eine solche quantitative Auswertung ist technisch möglich durch die Volltextsuche der Eighteenth Century Collections Online der Galegroup. Berücksichtigt wurde nur das politische, politisch-philosophische, juristische sowie historiographische Schrifttum. Dazu gehören auch die Periodika. Nicht berücksichtigt wurde das naturwissenschaftlich-medizinische sowie das theologische Schrifttum, um so einer Verwendungsweise des Krisenbegriffs im politisch-sozialen Kontext auf die Spur zu kommen.

${ }^{8}$ Also nicht im naturphilosophischen Sinne, was eine ältere Verwendungsweise ist, vgl. Anm. 9.

${ }^{9}$ Oxford English Dictionary, 2. Aufl.; vgl. dazu allg. David L. Smith, Art. Rudyerd, Sir Benjamin (1572-1658), in: Oxford Dictionary of National Biography, Oxford 2004; online edn, Jan 2008. [http://www.oxforddnb.com/view/article/24256, accessed 15 Nov 2008]

${ }^{10}$ Vgl. William Andrews: The astrological physitian. Shewing, how to finde out the cause and nature of a disease, according to the secret rules of the art of astrology. Also generall rules and instructions, teaching how to discover what part of the body is afflicted. With a perfect description of the diseases and infirmities, signified by the planets, in any of the twelve zodiacall constellations, together with a most exact method, shewing how to finde whether the sick shall live or dye, according to naturall causes; with an exact way how to finde the true crysis, judiciall or criticall days, London 1656; Nicholas Culpeper: Culpepers Semeiotica uranica: or, An astrological judgement of diseases from the decumbiture of the sick much enlarged. 1. From Aven Ezra by the way of introduction. 2. From Noel Duret by way of direction. Wherein is laid down, the way and manner of
} 
die politische Semantik diffundiert ist, sondern diese terminologische Verwandlung durchaus als bewusste Begriffsprägung durch sprachgewandte ,Politiker’ zu verstehen ist. So war es nach dem Poeten Rudyerd der dichtende Puritaner Benjamin Thompson (1642-1714), der damit, und zwar in Form eines Versepos, die Situation der Siedler in Neu-England im Konflikt mit den Indianern in den 1670er Jahren auf den Punkt brachte ${ }^{11}$. Ein anonymes Flugblatt befand wiederum, dass England 1689, also im Jahr der Glorious Revolution, in einer Krise gewesen sei $^{12}$. Tatsächlich begann Crisis nach der Revolution vermehrt in die politische Semantik einzudringen, und zwar wiederum als bewusste sprachliche Schöpfung: Jonathan Swift etwa beschrieb damit 1701 wortgewaltig die Folgen des Parteienstreits zwischen Whigs und Tories für England ${ }^{13}$. Die in den meisten ,Krisentexten’ des 18. Jahrhunderts gängige Formulierung „this present crisis“ kam schon in den 1703 wiederum von Swift edierten Briefen seines Mentors, dem 1699 verstorbenen Diplomaten und Essayisten Sir William Temple vor ${ }^{14}$. Solche Beispiele ließen sich leicht vermehren, was ich aber nicht tun möchte. Stattdessen will ich als einen ersten historischen Kontext für die Verwendung des Krisenbegriffs im 18. Jahrhundert die Kommentierung der Revolution von 1688/89 nennen. Bei tendenziell konservativen und den Tories nahe stehenden Autoren bildete Crisis nämlich einen - allerdings noch leisen - Kontrapunkt zu der bei den Whigs dominierenden Hochschätzung der Revolution als Werk göttlicher Vorsehung und als Wiederherstellung vermeintlich uralter Verfassungsverhältnisse ${ }^{15}$. Somit erhielt der Begriff eine parteipolitische Aufladung: Als Crisis bewertete man jeweils das, was die andere Seite als Fortschritt feierte. Umgekehrt konnten auch konservative Widerstände gegen Reformmaßnahmen als Weg in die Crisis bezeichnet werden. Als Daniel Defoe 1706 im Auftrag seines Patrons Robert Hartley um 1700 ein whiggistischer Spitzenpolitiker - Schottland bereiste, um für die Union mit

finding out the cause, change, and end of a disease. Also whether the sick be likely to live or die; and the time when recovery or death is to be expected. With the signs of life or death by the body of the sick party according to the judgment of Hippocrates. Whereunto is added, a table of logisticall logarithmes, to find the exact time of the crisis, London 1658; David de Planis Campy, Phlebotomiographia or, a treatise of phlebotomy.

Demonstrating the necessity of it in diseases; the time for elections. And likewise of the use and application of cupping-glasses, and leeches. Whereupon is added a brief and most methodicall tract of the crisis. Written originally in French, by Da de Plumis Campi chirurgion. And now faithfully rendred into English, by E.W. wellwisher to physick and chirurgery, London 1658.

${ }^{11}$ Benjamin Tompson, New Englands crisis, or, A brief narrative of New-Englands lamentable estate at present, compar'd with the former (but few) years of prosperity, Boston 1676; im gleichen Jahr auch in London erschienen.

${ }^{12}$ Anon., Englands crisis, or, The World well mended, London 1689.

13 Jonathan Swift, A discourse of the contests and dissensions between the nobles and the commons in Athens and Rome, with the consequences they had upon both those states, London 1701.

${ }^{14}$ Sir William Temple, Letters to the King, the Prince of Orange, the chief ministers of state, and other persons. By Sir W. Temple, Bart. Being the third and last volume. Published by Jonathan Swift, D.D., London 1703.

${ }^{15}$ Vgl. dazu John G.A. Pocock, The Ancient Constitution and the Feudal Law. A Study of English Historical Thought in the Seventeenth Century, Cambridge 1987 [zuerst 1957], 229ff. 
England zu werben, nannte er notorische parteipolitische und nationalistische Vorurteile gegen die Vereinigung Torheiten (follies), die zur Krise führten ${ }^{16}$. Die Unionsfrage erwies sich somit als ein zweiter begriffsgeschichtlicher Kontext von Krise im frühen 18.

Jahrhundert. Dies lag auch daran, dass die britische Union zentrale kirchenpolitische Fragen aufwarf, die ebenfalls im Medium des Krisenbegriffs diskutiert wurden ${ }^{17}$. Dass immer dann, wenn es um Fragen im Spannungsfeld von Politik und Religion ging, Crisis Konjunktur hatte, zeigte auch die Sacheverell-Affäre um 1710, die wir als einen dritten Kontext hervorheben wollen. Der Oxforder Theologe Henry Sacheverell hatte als Wortführer der den Tories nahe stehenden und die Revolution verdammenden ,High Church-Fraktion’ den Whigs vorgeworfen, England in eine presbyterianische Republik verwandeln zu wollen. Als man ihn deswegen vor Gericht stellte und am Ende nicht ihn, aber seine Schriften verbrannte, machte die Öffentlichkeit aus ihm einen politischen Märtyrer. In diesem Kontext versuchten sowohl Whigs als auch Tories die Situation als Krise zu klassifizieren ${ }^{18}$. Die infolge des Urteils in London ausgebrochenen Unruhen führten dabei zu einer besonderen Variante des parteipolitisch gebrauchten Krisenbegriffs, nämlich zur Kennzeichnung einer England existenziell bedrohenden jakobitischen Verschwörung ${ }^{19}$. Aus Sicht der Whig-Beobachter unerklärliche Phänomene wie die Londoner Unruhen - die Frage war ja, wie es sein konnte, dass ein von der Vorsehung erwähltes Volk plötzlich restaurative Tendenzen zeigte - wurden dunklen Machinationen der Stuart-Anhänger und der Katholiken insgesamt zugeschrieben. Damit erfüllte Crisis in der Publizistik des 18. Jahrhunderts jene Funktion, die noch im 17. Jahrhundert der Begriff Plot beziehungsweise Conspiracy besaß: Beide Begriffsverwendungen implizierten in vielen Fällen politisch motivierte Warnungen vor außergewöhnlichen, aber nicht näher definierten Gefahren für Staat und Gemeinwesen. Verbunden war mit beiden Begriffen dann stets die Aufforderung, radikale Entscheidungen und Schritte zu treffen und zu gehen. Zumindest der parteipolitisch gemünzte Krisenbegriff

\footnotetext{
${ }^{16}$ Daniel Defoe, An essay, at removing national prejudices, against a Union with England. Part III. By the author of the two first, Edinburgh 1706, 24; vgl. dazu Katherine R. Penovich, From „Revolution principles“ to Union. Daniel Defoe's intervention in the Scottish debate, in: A union for empire. Political thought and the British union of 1707, hrsg. von John Robertson, Washington DC 1995, 228-242.

${ }^{17}$ Sir Francis Grant, The patriot resolved. In a letter to an addresser, from his friend; of the same sentiments with himself; concerning the union, [Edinburgh] 1707, 15; Ders., An oration sacred to the imperial Majesty of Anne, Queen of Great-Britain, London 1707, 44.

${ }^{18}$ Henry Sacheverell, Collections of passages referr'd to by Dr. Henry Sacheverell in his answer to the articles of his impeachment [...], London 1710, 23; Gilbert Burnet, The Bishop of Salisbury his speech in the House of Lords, on the first article of the impeachment of Dr. Henry Sacheverell, London 1710, 10.

${ }^{19}$ Benjamin Hoadly, The Jacobite's hopes reviv'd by our late tumults and addresses: or, some necessary remarks upon a new modest pamphlet of Mr. Lesly's against the government, entituled, The good old cause: or, lying in truth, \&c., London 1710, 6.
} 
des 18. Jahrhunderts enthält daher latente Verschwörungstheorien ${ }^{20}$. Dies zeigt sich besonders deutlich an ,The Great Crisis' des Theologen Richard Roach von 1727, in dem Ereignisse wie Brände und unerklärliche Wetterphänomene als Krise bezeichnet und mit einer Verschwörungstheorie erklärt werden ${ }^{21}$. Eine Verschwörungstheorie steckte aber auch schon in Steeles ,The Crisis’ von 1714, in dem die Gefahr einer gewaltsamen, von Rom und den Jesuiten betriebenen Rekatholisierung Englands unter einem Stuart-Monarchen beschworen wurde - und das war seit den 1640er Jahren ein Topos in den Verschwörungsnarrativen. Steeles Pamphlet und vor allem die heftigen Reaktionen darauf waren im Übrigen der Grund dafür, warum im zweiten Jahrzehnt die Häufigkeit der Begriffsverwendung um rund 150\% zunahm. Steeles ,Crisis’ war allerdings zugleich Höhe- und Schlusspunkt der Diskussion der Revolution pro und contra im Medium des Krisenbegriffs. Denn die Thronfolge Georgs I. von Hannover 1714 erledigte bis auf weiteres die weit verbreiteten Sorgen, dass die Errungenschaften von 1688/89 schon wieder verloren seien. Ein anonymes Pamphlet konnte daher schon 1715 erleichtert von der Happy Crisis sprechen ${ }^{22}$.

Schon in den ersten beiden Jahrzehnten wurde es darüber hinaus auch üblich, außenpolitische Konjunkturen mit dem Krisenbegriff zu kommentieren - ein fünfter Kontext also, der für das 18. Jahrhundert höchst virulent war. So bezeichnete der Publizist Charles Davenant (16561714) damit die Situation Österreichs im europäischem Mächtekonzert schon um 1700: „Affairs are brought to such a Crisis that if the House of Austria is not in a Condition to oppose France, it cannot exist at all”. Die Politik Peters des Großen im Nordischen Krieg führte nach Auskunft eines Pamphlets von 1716 in eine Northern Crisis ${ }^{23}$. Richard Steele war es wiederum auch, der 1720 den Krisenbegriff zum ersten Mal im Zusammenhang mit einer ökonomischen Problemlage gebrauchte, als er nämlich die Folgen der berüchtigten ,South Sea Bubble’ für die Vermögensverhältnisse kommentierte (sechster Kontext). Im Übrigen waren die 1720 und 30er Jahre aber Jahrzehnte, in denen Politik, Religion und Gesellschaft kaum als krisenhaft beobachtet wurden. Dies zeigte sich auch am Rückgang der Krisensemantik in den

\footnotetext{
${ }^{20}$ Vgl. dazu vorerst Barry Coward / Julian Swann (Hrsg.), Conspiracies and conspiracy theory in early modern Europe. From the Waldensians to the French Revolution, Aldershot 2004; Umgekehrt bildeten Verschwörungen und Verschwörungstheorien im 17. Jahrhundert Krisenszenarien, und zwar nicht nur avant la lettre, vgl. etwa Michael F. Suarez, A Crisis in English Public Life. The Popish Plot, Naboth's Vineyard (1679), and MockBiblical Satire's Exemplary Redress, in: Huntington Library Quarterly, 67:4 (2004), 529-552.

${ }^{21}$ Richard Roach, The great crisis: or, the mystery of the times and seasons unfolded, with relation to the late disorder and confusion of the seasons of the year, and other signs of the times. With considerations and observations, [...] and some calculations of the numbers of time, [...] Part I., London 1725 [1727].

${ }^{22}$ Anon., The happy crisis: in a letter to the Right Honourable James Stanhope, Esq; one of His Majesty's principal secretaries of state, London 1715.

${ }^{23}$ Grefve Carl Gyllenborg, The northern crisis. Or, impartial reflections on the policies of the Czar, occasioned by Mynheer Von Stocken's reasons for delaying the descent upon Schonen [...], London 1716.
} 
ausgewerteten Textkorpora von 225 (1711-20) auf 150 (1721-30) beziehungsweise 200 (1731-40). Gerade in den 1720er Jahren wähnten sich die Briten bekanntlich in einem neuen ,augusteischen Zeitalter', in dem unter der Herrschaft des Premierministers Robert Walpole Frieden herrschte, der allgemeine Wohlstand wuchs, Großbritannien sein Kolonialreich erwarb und damit nicht zuletzt auch eine kulturelle Blütezeit einherging. Zwar wurde der Begriff ,Krise’ zwischen 1720 und 1740 durchaus verwendet, allerdings im Vergleich mit dem Jahrhundertbeginn mit diffusen Bezugspunkten. Dieser Trend setzte sich in den 1740er Jahren fort. Der Begriff Krise wurde auf alles Mögliche appliziert, jedoch nur sporadisch auf jenes Ereignis, wo eine Krisensemantik vielleicht zu erwarten gewesen wäre. Die Rede ist vom letzten Jakobitenaufstand 1746, der im Windschatten des Österreichischen Erbfolgekriegs stattfand $^{24}$. Der Enkel des letzten, 1688 vertriebenen Königs aus dem Hause Stuart, Charles Edward Stuart (1720-1788), war im Juli 1745 aus dem französischen Exil in Schottland zurückgekehrt. Gemeinsam mit den wichtigsten Highland Clans zettelte Charles einen Aufstand gegen das Haus Hannover und die regierenden Whigs an. In London und anderen Städten löste dieser Aufstand große Aufregung aus, weil die britischen Truppen zu diesem Zeitpunkt auf dem Kontinent gebunden waren und die Jakobiten immer weiter nach Süden vordrangen. Erst im April 1746 konnte der Aufstand niedergeschlagen werden. Statt crisis erlebte in diesem Kontext ein anderer moderner Grundbegriff seine semantische Neujustierung, nämlich terror ${ }^{25}$.Wie die quantitative Auswertung gezeigt hat, fällt das Gros der Begriffsverwendung in die zweite Jahrhunderthälfte. 1756, also am Beginn des Siebenjährigen Krieges, sahen die Publizisten eine Krise der britischen Macht in dem Fall gegeben, dass sich Krone und Parlament nicht auf ein energisches Vorgehen gegen Frankreich und eine Allianz mit Preußen verstünden (siebter Kontext) ${ }^{26}$. Dabei wurde freilich nicht allein der mögliche Verlust der politischen Position Englands in Europa als Krise geschildert, sondern vielmehr Einbußen beim britischen Kolonialreich in Nordamerika, in der Karibik und in Indien ${ }^{27}$. Der Tod Georgs II. 1760 und die Nachfolge Georgs III. 1761, also inmitten des Kriegs, wurden ebenso als Krisen geschildert wie seine schwere psychische Erkrankung

\footnotetext{
${ }^{24}$ Vgl. dazu Bruce Lenman, The Jacobite risings in Britain, 1689-1746, London 1980.

${ }^{25}$ Vgl. dazu demn. André Krischer: Verräter, Verschwörer, Terroristen. Juristische Klassifikationen, gesellschaftliche Wahrnehmungen und Visualisierungen von politischer Delinquenz und kollektiver Bedrohung in Großbritannien, 16.-19. Jahrhundert, in: Karl Härter / Beatrice de Graaf (Hg.), Polizei, politisches Verbrechen, ,Terrorismus’ und innere Sicherheit, Frankfurt a.M. 2009.

${ }^{26}$ Anon., The crisis, London 1756.

${ }^{27}$ Anon., Reflections on the welfare and prosperity of Great Britain in the present crisis, London 1756.
} 
$1788^{28}$ (achter und zehnter Kontext) ${ }^{29}$. Zu diesem Zeitpunkt hatten freilich schon der Abfall der amerikanischen Kolonien und die Unabhängigkeitserklärung der dreizehn Staaten zu einer bis dahin nie da gewesenen Intensität der Krisensemantik geführt (neunter Kontext). Diese sollte allerdings nur ein Jahr später durch den Diskurs über die Französische Revolution und ihre Folgen für Staat und Gesellschaft in Großbritannien noch übertroffen werden (zehnter Kontext). Den Schlusspunkt hinter die Geschichte der Krisensemantik im britischen 18. Jahrhundert setzten schließlich die Auseinandersetzungen mit der irischen Rebellion von 1795 (elfter Kontext).

Diese elf Kontexte können im Folgenden freilich nicht alle besprochen werden, und dies entspräche auch nicht der Fragestellung. Vielmehr soll es im Folgenden um die Erfindung von Crisis als Signalbegriff der politischen Publizistik gehen, die man dem erwähnten Richard Steele zuschreiben muss. Wenn im frühen 18. Jahrhundert von einer Krise die Rede war, dann vor allem in den Pamphleten, die als Quellengruppe und als Medium der Krisenbotschaft für meine Überlegungen eine besonders wichtige Rolle spielen. Crisis gehörte zu jenen terminologischen Zuspitzungen, mit denen Pamphletisten um Aufmerksamkeit für ihr Anliegen warben (Kapitel 2). In dieser medientheoretischen Hinsicht ersetzte der Begriff bei Steele nicht nur formal, sondern auch inhaltlich den um 1700 verblassten Begriff popery (beziehungsweise popish plot, popish conspiracy). Um diese semantische Erbfolge und die dem Begriff Crisis zumindest um 1700 noch inhärente Verschwörungstheorie geht es im dritten Kapitel. Das vierte und fünfte Kapitel fragen dann nach den publizistischen Verwendungsweisen von Crisis im Verlauf des 18. Jahrhunderts: Handelte es sich dabei um Indizien für ein kollektives Krisenbewusstsein oder nicht eher um Beispiele für den strategischen Gebrauch des Begriffs, um ein Mittel zur Durchsetzung politischer Interessen. Wie lassen sich diese Ebenen unterscheiden, und welche sprachgeschichtlichen Methoden bieten sich an, um die Begriffsverwendung in der Publizistik zu erklären?

\section{Richard Steeles ,The Crisis' - Pamphlete als Medium der Krisenbotschaft}

Richard Steeles Pamphlet ,The Crisis’, das am 26.12. 1714 in London erschien, bildete mindestens in zweifacher Hinsicht einen Paradigmenwechsel zur bis dahin ja bereits

\footnotetext{
${ }^{28}$ Anon., The crisis: or, considerations on the present state of affairs. London, 1761; Anon., Thoughts on the present alarming crisis. Humbly addressed to both Houses of Parliament. By a well-meaning Briton, London 1788.

${ }^{29}$ Vgl. dazu auch den Beitrag von Marian Füssel in diesem Band.
} 
gepflegten Krisensemantik ${ }^{30}$ : Zum einen prangte der Begriff hier zum ersten Mal auf dem Titelblatt (vgl. Abb. 1) - und man muss hinzufügen: nur auf dem Titelblatt, denn im Text selbst kam der Begriff nicht mehr vor. Zum anderen war überhaupt noch nie zuvor ein Pamphlet schon vor dem Erscheinen derart geschickt beworben und nach dem Erscheinen vermarktet und vertrieben worden. Die erste Auflage wurde in rund 40.000 Exemplaren gedruckt. Noch 1714 erschienen vier weitere Auflagen in ähnlicher Höhe sowie eine Übersetzung ins Französische ${ }^{31}$. Es ist nicht übertrieben, ,The Crisis’ als das publizistische Ereignis des Jahres 1714 zu bezeichnen, zumal dann nicht, wenn man die ebenfalls publizierten Reaktionen darauf berücksichtigt. Schon im Herbst 1714 hatte Steele den Text in seiner Zeitschrift ,Englishman’ angekündigt und für Subskriptionen geworben, die dann auch zahlreich einliefen. Auf diese Weise war es möglich, Drucker in London sowie in der Provinz davon zu überzeugen, das Pamphlet zu publizieren, denn die Nachfrage war ja im Vorhinein gesichert $^{32}$. Jonathan Swift, vor 1710 noch ein Freund Steeles, danach aber ein erbitterter Gegner, konnte sich den unerhörten Erfolg von ,The Crisis’ nur mit den geschickten Vertriebswegen des Pamphlets erklären, die er deswegen auch genau beschrieb: Demnach liefen um die Jahreswende 1714/15 Zeitungsjungen durch London und andere Städte und brüllten „The Crisis! The Crisis!“33. Bald sprach man in ganz London und anderen englischen Städten von The Crisis. Steele hatte es also geschafft, dass der Begriff Crisis buchstäblich in aller Munde war. Denn geradezu schlagartig war aus dem bislang eher distinguierten und nur pointiert eingesetzten Begriff der Gelehrten ein Allerweltswort geworden.

Richard Steele (1672-1729) wird traditionell zu den Gründungsfiguren des aufgeklärten Pressewesens gezählt. Zusammen mit seinem Kompagnon Joseph Addison publizierte er 1709-12 ,The Tatler' und ,The Spectator', 1713 dann ,The Guardian’ im Alleingang. Zu dieser Zeit war der Abgeordnete Steele bereits ein unerschütterlicher Whig - bereit, für seine Überzeugung, dass England in Gefahr sei, von einer französisch-katholischen Allianz erobert zu werden, auch Repressionen in Kauf zu nehmen. Steele wurde mehrfach wegen Aufwiegelung (sedition) angeklagt, wegen ,The Crisis’ wurde er sogar aus dem Unterhaus

\footnotetext{
${ }^{30}$ Zur gepflegten Semantik im Sinne der Üblichkeit einer Begriffsverwendung vgl. Niklas Luhmann, Gesellschaftsstruktur und Semantik. Studien zur Wissenssoziologie der modernen Welt, Bd. 1, Frankfurt a.M. 1980, $19 f$.

${ }^{31}$ Sir Richard Steele, La crise. Ou discours où l'on demontre par les actes les plus authentiques les justes causes de l'heureuse revolution. [...] Et quelques remarques, necessaires dans la conjoncture presente, sur le danger d'un successeur papiste. Par Mr. Richard Steele, ecuyer. Traduit de l'anglois par Mr. Boyer, London 1714.

${ }^{32}$ Vgl. George A. Aitken, The Life of Richard Steele, Bd. 2, London 1889, 3ff.

33 Jonathan Swift, The publick spirit of the Whigs, set forth in their generous encouragement of the author of the crisis. [...], London, 1714, 6.
} 
geworfen $^{34}$. Anders als die meisten Krisen-Pamphletisten des 18. Jahrhunderts, die anonym publizierten, bewies Steele jedoch Mut und stand zu seiner Veröffentlichung. In ihm jedoch wie Habermas einen Protagonisten der heraufziehenden bürgerlichen Öffentlichkeit zu sehen, der mit seinen Schriften und im sozialen Ort Kaffeehaus den kritischen Diskurs beförderte, wäre indes verfehlt. Der Historiker Brian Cowan hat kürzlich darauf hingewiesen, dass Steele in erster Linie Parteipropaganda betrieb: „it was to make the cultural politics of Augustan Britain safe for a Whig oligarchy“35. ,The Crisis’ griff vor allem den High Church Klerus an, dem Steele vorwarf, in der Frage der Hannoveraner Sukzession unzuverlässig zu sein und insgeheim an der Restauration des ,Pretenders’, also James Francis Stuart, zu arbeiten. Laut Steele bestand die Krise im wesentlichen in der ungelösten Frage, ob auf die kränkelnde Königin Anne der erhoffte protestantische König folgen würde, ob also der Act of Settlement von 1702 und alle anderen die Revolution sichernden Gesetze greifen würden oder nicht. Steele zitierte diese Gesetze in seinem Pamphlet im Wortlaut, weswegen Swift ihm vorwarf, im Grunde nichts anderes als ein schlecht ediertes Gesetzesblatt mit einigen eigenen Anmerkungen publiziert zu haben ${ }^{36}$. Trotzdem provozierte das Erscheinen von ,The Crisis' prompte Reaktionen. Ein anonymer Kleriker, der beanspruchte, für alle diejenigen zu sprechen, die Steele ja angegriffen hatte, fragte in seinen ebenfalls mehrfach aufgelegten ,Remarks on Mr. Steele's Crisis ${ }^{37}$, was der Autor denn eigentlich mit dem Titel meine: „Is it because this is the critical Minute for People to give into Fears and Jealousies concerning Administration of Affaires by the Queen and Present Ministry? Or are you induced to call it so from the Deference you are apt to pay to your own Opinion and Judgement? Are our National Disorders come to their Crisis, and our Groundless Discontents ripe enough for Mutinity and Rebellion, and Prescriptions for Phlebotomy, from you that have ursurp'd the Name of our Physician? [...]”38.

Man kann es als einen geschickten Schachzug des erfahrenen Publizisten Steele werten, dass er die Bedeutung des Begriffs Crisis völlig offen gelassen hatte und es somit seinen Gegnern aufgegeben war, über dessen Sinn zu rätseln. Den größten Erfolg erzielte Steele aber damit, dass das Dutzend an Gegenpamphleten darauf beharrte, es gebe weder in der einen noch in

\footnotetext{
${ }^{34}$ Nach der Hannoveraner Thronfolge wurde er allerdings als Held gefeiert und wieder als Abgeordneter zugelassen, vgl. zum Prozess 1714 Anon., The case of Richard Steele, Esq; being an impartial account of the proceedings against him. In a letter to a friend, London 1714.

${ }^{35}$ Brian William Cowan, Mr. Spectator and the Coffeehouse Public Sphere, in: Eighteenth-Century Studies, 37:3 (2004), 345-66, hier 361.

${ }^{36}$ Swift, The Publick Spirit (Anm. 29), 6.

${ }^{37}$ Anon., Remarks on Mr. Steele's Crisis, London 1714.

${ }^{38}$ Ebd., 4.
} 
der anderen Hinsicht eine Krise - und damit erst den Begriff als zeitdiagnostisches Medium anerkannte und publik machte ${ }^{39}$. Der Ärger darüber, dass es Steele gelungen war, mit einem solch suggestiven Begriff das politische Ansehen der Tories und der High Church angegriffen zu haben, durchzog alle Gegenpamphlete. Nicht zu unrecht warf man ihm zudem vor, mit diesem Begriff eine Art Marketingtrick benutzt zu haben, der nur den Verkauf in die Höhe treiben sollte ${ }^{40}$. Auch Swift vermutete, es sei Steele bei der Titelwahl nur um den Effekt, buchstäblich um eine Schlagzeile gegangen. Man solle doch mal die Leute fragen „whether they have read it? They will answer, No; but they have seen it everywhere, and it will do a World of Good: It is a Pamphlet, and a Pamphlet they hear against the Ministry, talks of Slavery, France, and the Pretender. They desire no more; it will settle the Wavering, confirm the Doubful, instruct the Ignorant, inflame the Clamorous, tho'it never be once looked into“41. Die Gefahr, die Swift von Steeles ,Crisis’ ausgehen sah, war also, dass allein der Titel bei ganz unterschiedlichen Personengruppen Anlass zu bestimmten Assoziationen geben würde: Die einen fühlten sich darin bestärkt, dass England und der Protestantismus bedroht seien, andere und weniger gut informierte würden sich erst zu dem Glauben verleiten lassen, dass dies so sei. Tatsächlich drehte sich die Kontroverse zwischen Steele und seinen Kritikern schon sehr bald gar nicht mehr um die Sache, sondern um die Begriffswahl. Es wurde nicht darüber diskutiert, ob es eine Krise gab oder nicht, sondern ob es zulässig war, eine politische Konstellation überhaupt so zu bezeichnen. Mit anderen Worten: es kam schon 1714 zu einem Metadiskurs über den Begriff der Krise als Zeitdiagnose. Als Steele publizistisch auf die Angriffe reagierte, behauptete er nicht mehr allein die Existenz einer Krise, sondern auch die Notwendigkeit zu einer solchen drastischen Wortwahl ${ }^{42}$. Dabei betonte er schließlich auch, dass er sehr wohl gezeigt habe, was er mit Krise meine: Nämlich das Gegenteil von liberty, health, strength und civil rights. Krise hingegen bedeute misery, Tyrannei, Not und Leid durch arbitrary power und a Popish Prince.

Dieser letzte Begriff, genauer Popish Successor, war allerdings auch schon auf dem Titelblatt von The Crisis zu lesen. Er war dort zudem durch eine Frakturschrift deutlich hervorgehoben.

\footnotetext{
${ }^{39}$ Anon., A letter to Mr. Steele, concerning his crisis. Edinburgh 1714; Anon., A speech suppos'd to be spoke by R---- St---l, Esq; at the opening this present Parliament, with some remarks in a letter to the bailiff of St---dge, very proper to be bound up with the Crisis, London 1714; Timothy Tomkins, A letter to Mr. Steele, concerning the removal of the Pretender from Lorrain, occasion'd by the Crisis. Written by an Englishman, London 1714.

${ }^{40}$ Anon., The false alarm: or, remarks upon Mr. Steele's crisis. Being a defence of the true constitution, and succession to the crown of England: and the case of the revolution fully considered, London 1714.

${ }^{41}$ Swift, The Publick Spirit (Anm. 29), 5.

${ }^{42}$ Sir Richard Steele, A defence of the crisis, written by Mr. Steele. Containing, a farther vindication of the late happy revolution. And the Protestant succession to the crown of England, in the illustrious house of Hanover, London 1714.
} 
Und was das für Folgen hatte, erkannte schon Swift: Wenn die Zeitungsjungen den Leuten das Pamphlet nämlich unter die Nase hielten, dann lese man wegen dieser visuellen Strategie des Titelblatts als erstes: The Crisis, und als zweites Popish Sucessor. Und schon sei klar, worum es ging: Denn wer auch nicht wusste, was er sich genau unter einer Crisis vorzustellen hatte, der wusste doch, was Popery hieß ${ }^{43}$. Denn dieser Begriff hatte in der englischen Pamphletistik bereits eine lange Tradition. Wir müssen daher an dieser Stelle einen kurzen begriffs- und auch mediengeschichtlichen Rückblick einschalten.

\section{Von Popery zu Crisis: Semantische Transformationen zwischen dem 17. und 18.}

\section{Jahrhundert}

Schon seit dem späten 16. Jahrhundert erfüllte der Begriff popery in den englischen Pamphleten eine bestimmte Funktion: Popery war im protestantischen Diskurs die AntiReligion schlechthin; das Fleisch und nicht das Geistige, das Äußere und Zeremoniöse, nicht das Innerliche und Wortorientierte. Popery bedeutete Tyrannei und nicht Freiheit (liberty) ${ }^{44}$, Dunkelheit und nicht Licht, Täuschung und nicht Offenbarung, Verdammnis und nicht Heil und so fort ${ }^{45}$. Durch den spanischen Angriff 1588 sowie diverse echte und fiktive Verschwörungen von Jesuiten und anderen Katholiken wurde popery begrifflich zudem mit Gewalt, Tod und Zerstörung von Außen aufgeladen, also von Seiten Spaniens und anderer katholischer Reiche ${ }^{46}$. Seit dieser Zeit war der Begriff ein typischer Bestandteil, geradezu ein essentieller Topos antikatholischer Pamphlete ${ }^{47}$. Die Geschichte des englischen Pamphlets und der Popery-Diskurs vollzogen sich um 1600 regelrecht koevolutionär ${ }^{48}$. Wie später Crisis durchlief auch Popery verschiedene begriffliche Konjunkturen, die wir hier im Einzelnen nicht besprechen wollen. Wichtig erscheint mir aber der Hinweis von Peter Lake, dass der Popery-Diskurs in den Pamphleten stets dann Hochkonjunktur hatte, wenn England im 17. Jahrhundert Krisen Phasen kollektiver Gefährdungen durchlief wie zum Beispiel die

\footnotetext{
${ }^{43}$ Vgl. dazu J.A. Downie, Public Opinion and the political pamphlet, in: The Cambridge History of English Literature. 1660-1780, hrsg. v. John Richetti, Cambridge 2005, 549-571, hier 558.

${ }^{44}$ Vgl. zum Begriff Quentin Skinner, Liberty before Liberalism, Cambridge 1998.

${ }^{45}$ Vgl. dazu grundlegend Peter Lake, Antipopery. The structure of a prejudice, in: Conflict in early Stuart England. Studies in religion and politics. 1603-1642, hrsg. v. Richard P. Cust / Ann Hughes, London 1989, 72106.

${ }^{46}$ Martina Mittag, National identity and the sovereign in anti-Spanish pamphlets 1558-1625, in: Costerus 137 (2001), 109-130.

${ }^{47}$ Ian Green, Print and Protestantism in early modern England, Oxford 2000.

${ }^{44}$ Jesse M. Lander, Inventing polemic. Religion, print, and literary culture in early modern England, Cambridge / New York 2006; Peter Lake / Michael C. Questier (Hrsg.), The Anti-Christ's lewd hat. Protestants, Papists and players in post-Reformation England, New Haven 2002.
} 
Pulverfassverschwörung von 1605 und vor allem den Bürgerkrieg 1642-48 ${ }^{49}$. Während der Begriff in diesen beiden Kontexten durchaus das Potential hatte, Identität zu stiften und die ,protestantische englische Nation’ von ,Anderen’ abzugrenzen, verlor er diese Macht spätestens während des so genannten Popish Plot 1679-82. Während dieser von den Whigs forcierten Verschwörungspanik, die auch das Ziel hatte, den katholischen Thronfolger James unmöglich zu machen, erlebte popery eine begriffliche Inflation. Zudem wurde das parteipolitische Interesse an diesem Begriff von der Gegenseite, der royalistischen ,Partei’ (den späteren Tories) und ihrem gewieften Kommunikationsexperten Robert L’Estrange erst enttarnt und dann ätzend ironisiert ${ }^{50}$. Warnten die Anhänger der Whigs in Pamphleten vor „The Growth of Popery“, dann antwortete L’Estrange darauf zum Beispiel mit einem Pamphlet mit dem Titel „The Growth of Knavery“ (Gaunerei). Die Jahre dauernde Verschwörungsphantasie wurde begleitet von einem bislang nicht gekannten Pamphletkrieg ${ }^{51}$, der auch zur Folge hatte, dass der Begriff popery seine traditionelle Signalwirkung verlor: Er mobilisierte seit der Mitte der 1680er Jahre keineswegs mehr kollektive Ängste, sondern provozierte Nachfragen, worauf sich der Verdacht denn eigentlich gründe. Dies war nun nicht allein die Folge einer völligen Überstrapazierung des Begriffs, sondern auch der Ausbildung einer neuen Form von medial konstituierter Öffentlichkeit, die zwar nur eingeschränkt das Prädikat ,kritisch’ erhalten kann ${ }^{52}$, aber zumindest doch nach differenzierteren Überzeugungsstrategien verlangte. Wir wollen davon ausgehen, dass diese Form der Öffentlichkeit 1714 noch im Wesentlichen die gleiche war. Nun lag es aber in der medialen Logik des Pamphlets, das im 18. Jahrhundert noch immer wichtiger werden sollte, mit bestimmten Signalen die Aufmerksamkeit des Lesers auf sich zu ziehen. Was schon Swift vermutet hatte, bestätigt nun die moderne Leseforschung: Pamphlete wurden, obwohl sie nie mehr als sechzig, gewöhnlich aber nur bis zu zehn Seiten hatten, nicht ,durchgelesen, ${ }^{53}$. Was vielmehr zählte, war die Titelseite, deren Layout so beschaffen sein musste, dass die zentrale

\footnotetext{
${ }^{49}$ Lake, Antipopery (Anm. 41), 83; ferner Robin Clifton, Fear of Popery, in: The Origins of the English Civil War, hrsg. von Conrad Russell, London 1973, 144-167.

${ }^{49}$ Mark Nicholls, Discovering Gunpowder Plot. The King's Book and the Dissemination of News, in: Recusant History, 28:3 (2007), 397-415; Jason Peacey, Politicans and pamphleteers. Propaganda during the English civil wars and interregnum, Aldershot 2004.

${ }^{50}$ Vgl. dazu demn. Peter Hinds, The Horrid Popish Plot. Roger L'Estrange and the Circulation of Political Discourse in Late-Seventeenth-Century, London / Oxford 2009; zuletzt ders., A Vast Ill Nature. Roger L'Estrange. Reputation, and the Credibility of Political Discourse in the Late Seventeenth Century, in: Seventeenth Century, 21:2 (2006), 335-363.

${ }^{51}$ Vgl. Joad Raymond, Pamphlets and pamphleteering in early modern Britain, Cambridge 2003, 323-382.

Dieser Pamphletkrieg wurde freilich durch den Kollaps der Zensurgesetze 1679 begünstigt.

${ }^{52}$ Dazu jetzt Mark Knights, How rational was the later Stuart public sphere?, in: The politics of the public sphere in early modern England (Politics, culture, and society in early modern Britain), hrsg. von Peter Lake / Steven C. Pincus, Manchester 2007, 252-267.

${ }^{53}$ Vgl. Raymond, Pamphlets (Anm. 47), 4ff.
} 
Botschaft auf einen Blick erfassbar wurde. Um 1700 leisteten dies zum einen stereotype Bilder $^{54}$ und zum anderen bestimmte Signalbegriffe. Die Signalwörter der Pamphlete befanden sich zudem stets in einer Art Überbietungswettbewerb, was allerdings im 18. Jahrhundert nicht mehr auf barocke Pleonasmen hinauslief wie noch zur Bürgerkriegszeit. Vielmehr ging es darum, mit einem möglichst prägnanten und zugespitzten, aber doch für Assoziationen offenen Begriff die Leser in den Bann zu schlagen.

Richard Steele war diese dem Medium Pamphlet geschuldete Zuspitzung mit dem Begriff Crisis offenbar in besonderer Weise gelungen. Dies war auch deswegen der Fall, weil er 1714 noch die semantische Brücke zu dem älteren Signalbegriff Popery geschlagen hatte, der die gleiche Botschaft transportiert hatte - nämlich die Warnung vor dem Ruin des Protestantismus und damit der englisch-britischen Nation. Bereits 1720 publizierte Steele ein weiteres Pamphlet mit dem ins Auge fallenden Titel ,The Crisis', diesmal allerdings ohne Popery-Bezüge. Sein Anliegen in ,The Crisis of Property' galt vielmehr den Folgen der Geschäfte des britischen Staats mit der South Sea Company. Diese war zu dieser Zeit durch den Sklaven- und Luxusgüterhandel in der Karibik eines der Vorzeigeunternehmen auf Aktien geworden. Um während dieser Boomphase nun noch weiter zu expandieren, vereinbarten das Unternehmen und die Regierung eine Umschichtung der Staatsschulden auf die Company; diese erhielt im Gegenzug das Recht, ihren Kapitalsockel anzuheben und darauf zusätzliche Aktien herauszugeben - letztlich also Spekulationen im großen Stil anzuheizen. Konkret sahen die Pläne vor, dass zehntausende Briten als Besitzer von Staatsanleihen - und diese waren eine wichtige Säule der britischen Finanzpolitik des 18. Jahrhunderts - nun nicht länger Gläubiger des Staats, sondern der South Sea Company waren. Die Renditen, die in Form von Jahrespensionen (annuities) ausgezahlt wurden, unterlagen damit faktisch den Gesetzen des Marktes $^{55}$. Steele nun erkannte ziemlich deutlich, dass in jenem Fall eine Vermögensvernichtung enormen Ausmaßes die Folge wäre, wenn die Geschäfte der Company doch nicht so rosig verlaufen sollten wie erhofft. Und nicht nur das: Sollte das South Sea Scheme der Regierung schief gehen, wäre auch ein ungeheuerer Vertrauensverlust in den Staat die Folge: „Credit is a belief that money is as safe [...] in the possession of another than in a man's own hands. [...] Any the least suspicion of national credit is the greatest wound a Government can receive”, warnte Steele ${ }^{56}$. Diese doppelte Vernichtung von - um mit

\footnotetext{
${ }^{54}$ Joseph Monteyne, The printed image in early modern London. Urban space, visual representation, and social exchange, Aldershot 2007.

${ }^{55}$ Dazu allg. John Patrick Carswell, The South Sea Bubble, Sutton 1993.

${ }^{56}$ Zit. nach Aitken, The Life of Richard Steele (Anm. 28), 240.
} 
Bourdieu zu sprechen - ökonomischem und symbolischem Kapital machte Steele zufolge die neuerliche Krise aus. Er prognostizierte daher in ,The Crisis of Property', wenn das Parlament dem Vorhaben zustimmen würde: „farewell the Wealth and Honour of Great Britain“ ${ }^{57}$. Wie schon 1714 nahmen seine Gegner - die nunmehr allerdings die Whigs selbst waren, darunter vor allem die Anhänger von Premierminister Walpole und Schatzkanzler John Aislabie Steeles Begriff auf: Ihm wurde - einmal mehr anonym - vorgeworfen, ein Beispiel für eine Crisis of Honesty darzustellen ${ }^{58}$. Steele würde das Publikum nämlich bewusst irreführen über die Absichten des Gesetzgebers ${ }^{59}$. Unglaubwürdig mache sich Steele dem Anonymus zufolge nun gerade deshalb, weil er den Begriff Crisis benutze, der viel zu übertrieben sei und von einem echten Gentleman vermieden würde. Crisis gehöre allenfalls zu den Phrasen von ,Libellous Pamphlets, ${ }^{60}$.

Den Zeitgenossen war die suggestive Macht des Begriffs Crisis also offenbar klar; ihn zu benutzen verletzte aber (angeblich) die ungeschriebene Etikette der Mediensprache und des öffentlichen Diskurses im frühen 18. Jahrhundert. Wie 1714 bewegte sich auch in diesem Fall die Krisen-Debatte auf einer Metaebene und drehte sich um die Frage, ob die Begriffswahl zulässig war oder nicht. Der anonyme Kritiker skizzierte dabei auch, was der Begriff tatsächlich bewirke, nämlich einen Skandal ${ }^{61}$. Steele versuche, den Gesetzgeber vor sich herzutreiben, indem er die Öffentlichkeit mit seiner Begriffswahl in Aufruhr versetze: „By this means, you alarm the Gentleman, the Soldier, and the Merchant, and endeavour to possess them, that all that is valuable amongst Men will be lost [...] “62. Die dem Begriff Crisis zugeschriebene Dynamik beruhte also darauf, dass eine im europäischen Vergleich überdurchschnittlich ausdifferenzierte Öffentlichkeit ${ }^{63}$ darauf sensibel reagierte und angestachelt werden konnte (,inflame the People against ther Representatives“664). Dieses letztlich populistische Moment lag in der Tat Steeles Begriffswahl zugrunde: man wird ihm nicht attestieren können, zum ersten Mal eine Finanzkrise als solche benannt und beschrieben

\footnotetext{
${ }^{57}$ Steele, The Crisis of Property, London 1720, 6.

${ }^{58}$ Anon., The crisis of honesty. Being an answer to the crisis of property. In a letter to Sir R---- S----, London 1720.

${ }^{59}$ Ebd., $7 f$.

${ }^{60}$ Ebd., 2.

${ }^{61}$ Was wiederum von besorgten Pensionären durchaus begrüßt wurde, vgl. Anon., Scandal no argument: an Oxford annuitant's letter to Sir Richard Steele, in answer to The crisis of honesty. With reasons why guardians should not expose their wards to sale, and pay their own debts out of their estates, being a short view of the South-Sea affair yet depending, London 1720.

${ }^{62}$ Anon., The crisis of honesty (Anm. 54), 14.

${ }^{63}$ Dazu zuletzt Peter Lake / Steven C. A. Pincus, Rethinking the Public Sphere in Early Modern England, in: Journal of British Studies 45:2 (2006), 270-292.

${ }^{64}$ Anon., The crisis of honesty (Anm. 54), 7.
} 
zu haben. Vielmehr zielte er darauf, den parlamentarischen Gesetzgebungsprozess in der Südsee-Sache dadurch aufzuhalten, dass er die Öffentlichkeit - und das hieß in dieser Zeit eben auch schon: die Wähler - gegen das Vorhaben aufbrachte. Dabei den von anderen Publizisten als anrüchig empfundenen Begriff Crisis zu benutzen, hielt er angesichts der ungeheuerlichen Bestechungen, mit denen die Regierung die Abgeordneten gefügig machte, nur für legitim. Steeles zweites Krisenpamphlet machte also bewusst außerparlamentarische Opposition, und dies in einer Zeit, in der ein laufendes Parlamentsverfahren immer noch als Arkanum behandelt wurde ${ }^{65}$.

\section{Crisis als Postulat im Mediendiskurs des 18. Jahrhunderts}

Mit ,The Crisis of Property' wurde nun schon eine wesentliche Funktion des Krisenbegriffs im 18. Jahrhundert vorgezeichnet: Im Laufe eines umstrittenen und noch nicht abgeschlossenen parlamentarischen Entscheidungsprozesses wurde versucht, eine bestimmte Position als die einzig richtige zu deklarieren. Alle anderen Alternativen wurden hingegen als sicherer Untergang der englischen beziehungsweise britischen Nation stigmatisiert ${ }^{66}$. Welche Position in der Krise die jeweils richtige war, um den Ruin zum Beispiel der britischen Freiheit, der Religion aber auch der Handelsinteressen abzuwenden, wurde in den Pamphleten vor 1750 bereits auf der ersten Seite mitgeteilt - und genau das entsprach, wie erwähnt, der kommunikativen Strategie dieses Mediums: So entdeckte ein William Revolution 1735, als die britische Konvenienz- und Balancepolitik infolge des polnischen Thronfolgekriegs ins Trudeln geriet, eine „real crisis: or, the necessity of giving immediate and powerful succour to the emperor against France and her present allies, in order to preserve the Balance of Power, our Trade, our Constitution, our Religion, and the Liberties of Europe “67. Bereits 1731 hatte ein Thomas English eine vergleichbare außenpolitische Krise ausgemacht und für den Gesetzgeber Entscheidungsbedarf gesehen, über dessen Inhalt er sich ein „impartial judgment“ erlaube: „the Fate of Europe may be conceiv’d to depend upon the Conduct of the British Parliament. From the Temperature and Spirit of that August Assembly will likewise come forth the particular Fate of our own Establishment and Constitution. Upon this Occasion, every Englishman has a Right to offer his Sentiments [...]”68. Wer eine Krise entdeckt hatte, war also gleichsam dazu aufgerufen, Mittel zur ihrer Überwindung zu

\footnotetext{
${ }^{65}$ Vgl. Jason Peacey, The Print Culture of Parliament. 1600-1800, in: Parliamentary History 26 (2007), 1-16.

${ }^{66}$ Vgl. zu dieser Semantik Koselleck, Kritik und Krise (Anm. 1), 105; Ders., Art. „Krise“, in: Geschichtliche Grundbegriffe, Stuttgart 2004, 617f.

${ }^{67}$ London 1735.

${ }^{68}$ Thomas English, The crisis: or, impartial judgment upon publick affairs, London 1731.
} 
benennen. Das Krisenpostulat verband sich deswegen mit einem Anspruch auf Partizipation am politischen Entscheidungsprozess. Einige der Krisen-Pamphletisten waren ja selbst Abgeordnete, die sich zum einen den Mehrheitsverhältnissen entgegenstellten. Dabei vertraten sie im Schutz der Anonymität gewöhnlich Positionen, die sie persönlich im Parlament nicht vertreten konnten, ohne ihre Karriere zu riskieren. Doch nicht alle Pamphletisten betrieben Opposition. So konnte zum anderen auch eine sich bereits abzeichnende Entscheidung als die einzig richtige angesichts der Crisis präsentiert werden. Pamphletisten ohne Parlamentssitz waren von einer solchen Affirmation jedoch gewöhnlich weit entfernt. 1733 skizzierte ein Anonymus unter dem Titel ,The Crisis’ die „fatal consequences that will certainly ensue from [...] the late projected excise scheme”, also einer Steuererhöhung ${ }^{69}$. Ein anderer anonymer Autor sah 1756 ohne den Entschluss zum Krieg gegen Frankreich eine fatal crisis $^{70} .1766$ sah ein Pamphlet das britische Kolonialreich in der Krise, wenn der Stamp Act, also die Besteuerung sämtlicher Drucksachen in den amerikanischen Kolonien - wirklich durchgesetzt würde ${ }^{71}$. Als 1788 die Geisteskrankheit Georgs III. allgemein bekannt wurde und der König nicht länger in der Lage war, seinen Amtspflichten nachzukommen, galt dies einem „well-meaning Briton“ als eine „present awful“ und sogar „alarming crisis“72. Wie war diese zu überwinden? „How we can do it, I can not say, but I am certain I can say, how we can not do it, and that is, by appointing a Council of Regency““73. Ein solcher Regentenrat würde nämlich als erstes Premierminister William Pitt d.J. vom Amt entheben und dadurch alles nur noch schlimmer machen. Vor dem zweiten Koalitionskrieg gegen die französische Republik 1798 konstatierte der Diplomat Frederick Howard, Earl of Carlisle, eine „Crisis and its Alternatives“: „The Crisis, to which we are now brought, presents to our choice the two alternatives of Ruin and Salvation. No third alternative can possibly have place; and one of those two is unavoidably hanging over us“" ${ }^{\text {74 }}$. Gemeint war mit diesen greifbaren Alternativen freilich der erneute Krieg gegen Frankreich.

\footnotetext{
${ }^{69}$ Anon., The crisis: or, the Briton's advocate, London 1733.

${ }^{70}$ Anon., Reflections on the welfare and prosperity of Great Britain (Anm. 24).

${ }^{71}$ Anon., The crisis. Or, a full defence of the colonies. In which it is incontestibly proved that the British constitution has been flagrantly violated in the late Stamp Act, and rendered indisputably evident, that the mother country cannot lay any arbitrary tax upon the Americans, without destroying the essence of her own liberties, London 1766.

${ }^{72}$ Anon., Thoughts on the present alarming crisis. Humbly addressed to both Houses of Parliament. By a wellmeaning Briton, London 1788.

${ }^{73}$ Ebd., 10.

${ }^{74}$ Frederick Howard Earl of Carlisle, The crisis; and its alternatives offered to the free choice of Englishmen. Being an abridgment of „Earnest and serious reflections on the urgency of the present crisis, \&c.“, London 1798, 4.
} 
Das Postulat einer Krise, so zeigen diese Beispiele, ging in der Pamphletistik des 18. Jahrhunderts also jeweils einher mit Ratschlägen, die abzulehnen angesichts dieser Zeitdiagnose ein Fehler mit existenziellen Folgen für Großbritannien wäre. Aus der KrisenDiagnose, das zeigt besonders das letzte und durchaus typische Beispiel von 1798, ergaben sich ,Handlungszwänge’, die angeblich kein Abwägen, kein Aussitzen und schon gar keinen dritten Weg erlaubten. Die in der Krise jeweils zu treffenden Entscheidungen wurden als schiere Notwendigkeit stilisiert. Wenn eine Krise, wie Koselleck gezeigt hat, Kontingenz offenbart, dann beanspruchen die in den Krisenpamphleten skizzierten Programme jeweils den einzig richtigen Weg, um das Leben wieder in die gewohnten Bahnen zu lenken. Es wäre noch genauer zu zeigen, aber es scheint so, als ob Crisis als Postulat damit zu jenen semantisch konstituierten ,Sachzwängen’ gehörte, die die politischen Handlungsspielräume in Großbritannien am Ende des 18. Jahrhunderts diskursiv absteckten ${ }^{75}$.

\section{Verfügbares und Unverfügbares in der Krise}

Bereits in den 1720er Jahren konnte Crisis publizistisch verwendet werden, ohne eine Metadebatte über den Begriff zu eröffnen wie in Steeles Fall. Crisis avancierte im publizistischen Diskurs vielmehr zu einem neuen Paradigma ${ }^{76}$ und gewann als Begriff den Status diskursiver Üblichkeit. Seit der Jahrhundertmitte fungierte Crisis zudem auch nicht mehr allein als Signalbegriff auf dem Titelblatt eines Pamphlets, sondern wurde schließlich zum Topos in der Argumentation selbst. Dies änderte aber nichts daran, dass es sich dabei in vielen Fällen weiterhin um ein Postulat handelte und nicht unbedingt um eine kollektive Zeitdiagnose wenn eine Krise postuliert wurde, dann gingen die Publizisten auch davon aus, dass Mittel zur Verfügung standen, um die Krise zu überwinden. In diesem Sinne galt eine Krise auch als Chance - eine Konstellation, die die Publizisten als Happy Crisis bezeichneten ${ }^{77}$. Als Georg II. 1761 während des Siebenjährigen Kriegs plötzlich verstarb, postulierte ein Pamphletist ausdrücklich die Existenz einer Krise ${ }^{78}$, warnte aber davor, in Angst zu verfallen: „Fear is ill-grounded. We will therefore endeavour to shew, that our Publick Affaires are not in that dangerous State [...] and that they are not only Glorious, but

\footnotetext{
${ }^{75}$ Vgl. dazu Willibald Steinmetz, Das Sagbare und das Machbare. Zum Wandel politischer Handlungsspielräume in England 1780-1867 (Sprache und Geschichte, 21), Stuttgart 1993; vgl. dazu auch den Beitrag von Philip Hoffmann in diesem Band.

${ }^{76}$ Im Sinne der Wissenschaftstheorie von Thomas S. Kuhn; vgl. allerdings zu den Problemen der Applikation dieser Theorie im Kontext diskursgeschichtlicher Analysen Andreas Pećar / Kai Trampedach, Der „Biblizismus“ - eine politische Sprache der Vormoderne?, in: Die Bibel als politisches Argument, hrsg. v. dies., München 2007, 1-18, hier 6f.

${ }^{77}$ Anon., Reflections on the welfare and prosperity of Great Britain (Anm. 24), 49.

${ }^{78}$ Anon., The crisis: or, considerations on the present state of affairs (Anm. 25).
} 
Hopeful“79. Hoffnung sei gegeben, wenn nun nicht die Fraktion der Sparer obsiege: „Victory should be purchased with Money, and not Money saved at the Expence of Victory“، ${ }^{\circ 0}$.

Die bisher untersuchten Texte sind dadurch gekennzeichnet, dass sie ein deduktives Krisenkonzept präsentieren: Eine bestimmte Sachlage wird als Crisis gekennzeichnet, was dann mehr oder weniger umstritten war. Aus diesem Postulat wurden dann Maßnahmen abgeleitet, um der Crisis beizukommen. Es liegt auf der Hand, dass aus dieser Begriffsverwendung, die im Laufe des 18. Jahrhunderts exponentiell zunahm, nicht unmittelbar Rückschlüsse auf ein allgemeines Krisenbewusstsein zu gewinnen sind. Der Begriff Crisis wird vielmehr als politisches Instrument eingesetzt, um die Durchsetzung bestimmter Interessen gerade auch semantisch zu forcieren. Fragt man nach theoretischen Konzepten, um diese Begriffsverwendung genauer zu erfassen, dann bieten sich weniger die klassische Begriffsgeschichte, noch weniger die Diskursanalyse, sondern vor allem die sprachpragmatische Analyse der Cambridge School um Quentin Skinner und John Pocock $\mathrm{an}^{81}$. Das Postulat einer Krise war demnach eine politische Handlung „with words“. Im Sinne eines performativen Akts stellte die Konstatierung einer Krise keine ,wahre’ oder ,falsche’, sondern vielmehr eine gelungene oder misslungene Zeitdiagnose dar. Wem es gelang, eine bestimmte politische oder ökonomische Konstellation öffentlich als Krise zu klassifizieren, der konnte daraus bestimmte Konsequenzen ableiten, und vor allem darauf kam es an. Die gehäufte und schließlich ubiquitäre Begriffsverwendung in der zweiten Jahrhunderthälfte folgte der Logik einer ,politischen Sprache', deren Referenzpunkte zwar noch genauer zu bestimmen sind, die aber darauf beruhte, mit extremen Alternativen zu argumentieren (ruin/salvation, our posterity may live/national death). Crisis als Element einer politischen Sprache zu betrachten heißt davon auszugehen, dass der Begriff im politischen Diskurs bewusst als eine semantische Möglichkeit unter mehreren gewählt wurde, als ein performativer Sprechakt eingesetzt wurde, weil sich der Autor/Sprecher davon maximalen Nutzen für sein Anliegen versprach. Wie Andreas Pećar gezeigt hat, ist das Sprechen einer politischen Sprache mit dem Spielen einer sozialen Rolle vergleichbar; es ist aber nicht unbedingt die Konsequenz eines sozialen Habitus im Sinne von Bourdieu ${ }^{82}$.

\footnotetext{
${ }^{79}$ Ebd., 2.

${ }^{80}$ Ebd., 27.

${ }^{81}$ Vgl. Pećar / Trampedach, Der „Biblizismus“ (Anm. 73), 4; allg. dazu Eckhart Hellmuth / Christoph von Ehrenstein, Intellectual History made in Britain. Die Cambridge School und ihre Kritiker, in: Geschichte und Gesellschaft 27 (2001), 149-172. Zur Methode vgl. Quentin Skinner, Visions of Politics, Bd. 1: Regarding Method, Cambridge 2002.

${ }^{82}$ Pećar / Trampedach, Der „Biblizismus“ (Anm. 73), 5.
} 
Gab es also im britischen 17. Jahrhundert trotz der massenhaften Begriffsverwendung kein wirkliches Krisenbewusstsein? Schon Koselleck bemerkte ja: „Wenn der gehäufte Wortgebrauch ein hinreichendes Indiz für eine wirkliche Krise wäre, dann müssten wir in einer allumfassenden Krise leben. Aber dieser Rückschluss zeugt zunächst mehr von einer diffusen Redeweise, als dass er schon zur Diagnose unserer Lage beitrüge“ ${ }^{83}$. Die Frage, wo Crisis als Vokabel einer politischen Sprache aufhörte und stattdessen eine kollektive Wahrnehmung auf den Begriff brachte - und somit eben doch habituell verwendet wurde -, lässt sich zumindest nicht eindeutig beantworten. Dazu reichen die hier zugrunde gelegten Quellen zudem nicht aus, es müssten auch Selbstzeugnisse, Briefe usf. ausgewertet werden. Ich vermute allerdings, dass die regelmäßige strategische Verwendung des Begriffs in der Publizistik nicht nur zu seiner Implementation in die englische Alltagssprache führte, sondern auch zum Glauben an das, was beschworen wurde. Dem Zitat zufolge schien Koselleck noch von einer Diskrepanz zwischen Sprache und Wirklichkeit auszugehen, wobei Sprache die Wirklichkeit missverstehen oder falsch darstellen kann. Eine Prämisse der neueren Kulturgeschichte lautet demgegenüber, dass soziale Wirklichkeit nicht unabhängig von ihrer sprachlichen Konstitution existiert und alle soziale Realität letztlich Kommunikation ist ${ }^{84}$. Sprache stellt nicht nur Wirklichkeit dar, sondern auch her. Insofern ist es nicht sinnvoll, die englische Begriffsgeschichte der Krise allein danach zu unterteilen, wo Crisis entweder strategisch oder diagnostisch verwendet wurde. Wie lässt sich schon ausschließen, dass ein Pamphletist, der mit dem Crisis-Balken auf dem Titelblatt auf höhere Absätze hoffte, nicht auch an die von ihm postulierte Krise geglaubt hat? Wie das Spielen einer sozialen Rolle zudem allmählich zur ,zweiten Haut', zum Habitus werden kann, so stellte die permanente und ubiquitäre Verwendung des Krisenbegriffs am Ende des 18. Jahrhunderts zumindest ein Indiz für die kollektive Akzeptanz dieser Zeitdiagnose dar. Als Möglichkeit zur Annäherung an die freilich mediale Repräsentation des Krisenbewusstseins kann allerdings die Frage dienen, wo Crisis nicht mehr tautologisch oder als Pleonasmus, sondern als „nichtersetzbarer Grundbegriff“ (Koselleck) gebraucht wurde, was hier aber nicht mehr geleistet werden kann. Eine weitere Annäherung ermöglichen wiederum jene Texte, die die Krise als etwas nicht Beherrschbares schildern, und darauf möchte ich abschließend noch eingehen.

\footnotetext{
${ }^{83}$ Reinhard Koselleck, Begriffsgeschichten. Studien zur Semantik und Pragmatik der politischen und sozialen Sprache, Frankfurt a.M. 2006, 203.

${ }^{84}$ Vgl. dazu allg. Barbara Stollberg-Rilinger, Symbolische Kommunikation in der Vormoderne. Begriffe Forschungsperspektiven - Thesen, in: Zeitschrift für Historische Forschung 31 (2004), 489-527.
} 
Wie erwähnt, gingen die meisten Pamphletisten davon aus, dass eine Krise mit radikalen Maßnahmen (Kriegseintritt, Steuern erhöhen/senken und so fort) letztlich doch überwunden werden könne und in diesem Zusammenhang auch eine Chance für prinzipielle Neuerungen sei. Es gab jedoch auch Fälle, in denen mit der Beobachtung einer Krise keine Mittel genannt wurden, um über diese zu verfügen, die Krise somit permanent zu werden drohte. Die Begriffsverwendung erfolgte in jenen Texten, die die Krise als etwas Unverfügbares setzen, induktiv: Bestimmte Ereignisse und Strukturen wurden geschildert und im Begriff der Krise verdichtet, ohne dass Auswege erkennbar wären. In diesem Sinne anerkannte bereits 1736 ein Anonymus die Realität des Parteiendualismus als permanente Krise: „Party Spirit had exasperated the Country, Party Spirit had inflamed the Court [...] the publick Virtue of Patriotism was lost in the private Passion of Party [...] Party Names had been invented to encourage Division and Violence. Men conversed only with those of their Denomination, and hated every one else [...] Thus the Nation was divided worse than in Civil War“ ${ }^{85}$. Ein Rezept gegen den verderblichen Party Spirit wusste der Autor indes nicht. Das Problem ließ sich nur feststellen, nicht beheben: „The History of Britain can hardly instance a Crisis of greater and more general Importance, than the present Period of Time“" ${ }^{\text {86 }}$. Wenn aus der Beobachtung der Krise keine logischen und notwendigen Entscheidungen zu ihrer Überwindung abgeleitet werden können, dann wurde die Krise permanent, pathologisch und führte schließlich zur individuellen und kollektiven Melancholie angesichts der Zeitläufe ${ }^{87}$. Im besonderen Maß beherrschte ein melancholisches Krisenbewusstsein die Pamphlete im Kontext des amerikanischen Unabhängigkeitskriegs in den späten 1770er und frühen 1780er Jahren ${ }^{88}$. Die Loslösung der Kolonien und die Gründung der Vereinigten Staaten wurden als irreversible Prozesse, als historische Tatsachen erkannt, die Großbritannien und seinen imperialen Anspruch in die Krise führten ${ }^{89}$. In diesem Zusammenhang schob sich die zeitdiagnostische Dimension des Begriffs besonders auffällig in den Vordergrund: Mit der amerikanischen

\footnotetext{
${ }^{85}$ Anon., Remarks upon the present crisis. Humbly address'd to the knights, citizens and burgesses, to serve in the present Parliament, London 1736, 5f.

${ }^{86}$ Ebd., 4.

${ }^{87}$ Das 16. und 17. Jahrhundert hatte in diesem Zusammenhang bekanntlich Utopien entworfen, vgl. dazu Wolf Lepenies, Melancholie und Gesellschaft, Frankfurt a.M. 1969; Belege für den Zusammenhang unüberwindlicher Krise und Melancholie: Anon., Remarks upon the present crisis (Anm. 82), 5; Peter Williamson, Some considerations on the present state of affairs. Wherein the defenceless situation of Great-Britain, is pointed out [...], York 1758, 7; John Douglas, Seasonable hints from an honest man on the present important crisis of a new reign and a new parliament, Dublin 1761, 3, 14, 16, 44.

${ }^{88}$ Vgl. dazu jetzt British pamphlets on the American Revolution, 1763-1785, hrsg. von Harry T. Dickinson, London 2007.

${ }^{89}$ Dabei sind im Kontext der amerikanischen Revolution freilich verschiedene Krisensemantiken zu unterscheiden: Zum einen solche, die damit die Probleme der britischen Kolonialpolitik zu fassen versuchten, zum anderen jene, die mit Crisis den „Vollzug eines moralischen Gerichts“ meinten, den „Sieg der Freiheit und Unschuld“ wie Thomas Paine, vgl. Koselleck, Kritik und Krise (Anm. 1), 152.
} 
Revolution verlor der Krisenbegriff nämlich seine in der britischen Publizistik bereits traditionell instrumentelle Funktion als Mittel zur Durchsetzung bestimmter politischer Entscheidungen. Es ging bei der Begriffsverwendung nicht länger allein um Alternativen innerhalb eines bestimmten Handlungsrahmens (Krieg oder Frieden, Steuern oder Entlastung). Crisis wurde vielmehr zum Medium kollektiver, letztlich nationaler Selbsterkenntnis angesichts schon nicht mehr existenter Handlungsspielräume in der amerikanischen Frage, was dazu zwang, zu neuen Ufern aufzubrechen, buchstäblich nach Indien und sinngemäß als Handelsmacht. 1785 diagnostiziert zum Beispiel ein Thomas Brooke Clarke mit Blick auf das Ende des Unabhängigkeitskriegs: „Britain is arrived at a Crisis, whence she must experience a destiniy of exaltation or abasement“ ${ }^{\text {90 }}$. Der „decline of National Importance“ könne nicht mehr durch einen Sieg in Nordamerika, sondern allein durch die Konzentration auf Industrie und Handel aufgehalten werden ${ }^{91}$. Zwar generierte die Feststellung der Krise auch bei Clarke die Notwendigkeit zur Entscheidung, aber nicht mehr zwischen zwei klaren Alternativen. Es ging vielmehr um die Rückbesinnung auf nationale Stärke und auf Tradition, um somit weitere Erniedrigung (abasement) zu verhüten und zum Aufbruch in eine verheißungsvolle Zukunft (exaltation) zu ermutigen. Wenn die Publizistik der 1780er Jahre eine Krise feststellte, dann verzichtete sie auf das Drängen nach schnellen Entscheidungen wie in den Jahrzehnten zuvor. Vor politischen Entscheidungen in der Krise zur Neugestaltung der Zukunft stand die Rückbesinnung auf die eigene Geschichte als Horizont und Erfahrungsraum möglichen Handelns. Clarke schrieb: „While we are reasoning on those future events, we shall call in the aid of past and the guidance of present times. And while we are consulting for Britain, our views shall be extended over the Universe" ${ }^{92}$. Ein John Williams empfahl die eigene Nation und deren Geschichte sogar als Quelle des Trostes für jeden Engländer und Rückhalt bei der Neuorientierung: „At this crisis, every good citizen should look upon his country, as the sympathetic Samaritan look'd at and reliev'd the affliction of even a stranger" ${ }^{93}$.

\section{Fazit}

Nach einigen Vorläufern im 17. Jahrhundert wurde der Begriff Crisis in der politischen Publizistik Großbritanniens vor allem im parteipolitischen Diskurs nach der Revolution von

\footnotetext{
${ }^{90}$ Thomas Brooke Clarke, The crisis; or, immediate concernments of the British Empire, London 1785, 1.

${ }^{91}$ Ebd.

${ }^{92}$ Ebd., 1.

93 John Williams, The present crisis of the colonies considered; with some observations on the necessity of properly connecting their commerical interest with [...] Second edition, London 1786, viii.
} 
1688/89 virulent. Aber erst der Publizist Richard Steele etablierte 1714 den Begriff Crisis in einem so betitelten Pamphlet als Schlagwort im öffentlichen Diskurs. Mit der

Begriffsverwendung verband sich bei Steele aber keineswegs eine ausgefeilte Zeitdiagnose. Vielmehr nutzte er Crisis als Absatz fördernden und auf den Effekt zielenden Signalbegriff. In der politischen Pamphletistik trat Crisis das Erbe des älteren Signalbegriffs Popery an, zu dem Steele 1714 noch die Brücke geschlagen hatte. Die Briten besaßen um 1700 ein gewisses Vorverständnis über die Bedeutung von Crisis, was nicht nur die Funktionsweise des Signalbegriffs erklärt, sondern auch die Debatte um die Zulässigkeit dieser Begriffsverwendung, die sich jeweils an Steeles Krisenpamphlete von 1714 und 1720 anschloss. Letztlich trug diese Debatte aber nur zur weiteren Popularisierung des Begriffs bei. Schon vor der Jahrhundertmitte deutete sich allerdings an, dass der Krisenbegriff in der politischen Publizistik nicht nur üblich wurde, sondern auch seine Virulenz nicht mehr aus der Tradition speiste. Crisis wurde in den Pamphleten vielmehr zu einem Postulat, mit dem bestimmte politische Entscheidungen forciert oder auch nachträglich sanktioniert werden sollten. Mit der Verwendung des Begriffs gingen bestimmte Interessen einher - und das Gespür dafür, gerade auf diese Weise spezifische Entscheidungen forcieren zu können. Als Indikator für ein Krisenbewusstsein, oder besser gesagt: für die kollektive Beobachtung einer bestimmten Situation als Krise, kann die gehäufte Begriffsverwendung ab 1750 indes nicht unbedingt herhalten. Gleichzeitig wurde es für Publizisten aber leichter als noch für Steele, bestimmte politisch-soziale Konstellationen als Krise zu bezeichnen, Metadebatten über die Zulässigkeit der Begriffswahl wie noch 1714 blieben aus. Es kam auch trotz der zuletzt massenhaften und ubiquitären Verwendung von Crisis nicht zu einer semantischen Inflation und Entwertung des Begriffs wie im Falle von Popery. Dies scheint damit zusammenzuhängen, dass Crisis überwiegend nicht im Sinne einer wahren oder falschen Zeitdiagnose verwendet wurde, die dann minutiös als zutreffend oder unzutreffend nachgewiesen werden musste. Der Erfolg des Begriffs Crisis im politisch-sozialen Diskurs und dies gilt noch für die Krisensemantik des frühen 21. Jahrhunderts - gründete sich darauf, dass er immer dann und solange verwendet wurde, wenn und wie dies öffentliche Resonanz versprach. Die Begriffsverwendung stand unter den Bedingungen des Gelingens, was Crisis im publizistischen Diskurs als performativen Akt, letztlich als semantisches Ritual ausweist. Anders als popery und popish conspiracy, die Subsidiärbegriffe von Krise im 17. Jahrhundert darstellten, ließ sich eine postulierte Krise nicht mit polizeilichen und geheimdienstlichen Mitteln als Täuschung aufdecken. Die Behauptung einer Krise war nicht eindeutig falsifizierbar. Erst am Ende des 18. Jahrhunderts und infolge der faktischen Unabhängigkeit 
der Vereinigten Staaten von Amerika lässt sich auch in der britischen Publizistik eine Krisensemantik nachweisen, die eher auf kollektive Selbsterkenntnis gerichtet ist, auf die Frage, wohin die Zukunft gehen kann, wenn die Geschichte einen irreversiblen Verlauf genommen hat. Diese diagnostische, retrospektive und dann therapeutische Krisensemantik ist der Punkt, an dem Koselleck den Beitrag des Begriffs Krise zur „Pathogenese der bürgerlichen Welt“ ausmacht. Es sollte jedoch am Beispiel der politischen Publizistik Großbritanniens des 18. Jahrhunderts deutlich geworden sein, dass der Begriff keineswegs allein zum Arsenal von aufgeklärter Öffentlichkeit und bürgerlicher Emanzipation gehörte. Er war vor allem ein Medium, mit dem politische Deutungshoheiten erobert und auf rasche Entscheidungen gedrungen werden sollte. Jenseits feiner Differenzierungen war er nicht zuletzt ein semantisches Instrument, mit dem die Aufmerksamkeit auf ein bestimmtes Pamphlet gelenkt werden sollte, was sich in letzter Konsequenz somit auch als Marketingstrategie erwies. 